\title{
Highlights from ATLAS
}

\author{
L. Bellagamba ${ }^{1, a}$ on behalf of the ATLAS Collaboration \\ ${ }^{1}$ Istituto Nazionale di Fisica Nucleare, Bologna
}

\begin{abstract}
This report presents an overview of some of the most recent results obtained by the ATLAS Collaboration using $p p$ and heavy-ion collisions at the LHC. The review is not intended to be comprehensive and includes recent updates on the Higgs boson properties, precision Standard Model measurements, as well as searches for new physics. Most of the results exploit the data collected in the last LHC run, providing $p p$ collisions at a centre of mass energy of $13 \mathrm{TeV}$.
\end{abstract}

\section{Introduction}

Since the beginning of the Large Hadron Collider (LHC) data-taking, ATLAS [1] and the other experiments produced an impressive amount of results. Beside the discovery of the Higgs boson, which was the last missing particle of the Standard Model (SM), a large number of measurements tested all the SM sectors with unprecedented precision and the investigation for possible new physics signals has been pushed to domains far beyond the reach of the pre-LHC era. This report presents a compilation, which represents a personal selection and is not intended to be comprehensive, of relevant ATLAS results, with a special focus on fresh studies mostly performed with data collected in the last LHC run, providing $p p$ collisions at a centre of mass energy $(\sqrt{s})$ of $13 \mathrm{TeV}$. All this remarkable scientific production would not have been possible without the exceptional reliability of the LHC operations, that allow both ATLAS and CMS collaborations to collect an integrated luminosity of data close to $30 \mathrm{fb}^{-1}$ in Run 1 at $\sqrt{s}=7$ and $8 \mathrm{TeV}$ and close to $40 \mathrm{fb}^{-1}$ (up to 2016) in Run 2 at $\sqrt{s}=13 \mathrm{TeV}$.

\section{Higgs physics}

After the discovery of the Higgs boson $(H)$, which was mainly based on the di-photon and four-leptons final-states, a lot of progress has been done in studying the properties of the new boson in different production and decay channels.

\subsection{Higgs decay to $b$-quark pairs}

The dominant decay channel in the SM picture is the $b \bar{b}$ final state, with a branching ratio close to $60 \%$ but experimentally very challenging due to the overwhelming QCD background in the main gluon-gluon fusion production process. The ATLAS experiment performed a search for this decay

\footnotetext{
ae-mail: lorenzo.bellagamba@bo.infn.it
} 
using data collected in 2015 and 2016 at $\sqrt{s}=13 \mathrm{TeV}$ and corresponding to an integrated luminosity of $36 \mathrm{fb}^{-1}$ [2]. In order to improve the signal to background ratio the search has been performed in the associated production of the $H$ with a weak vector boson $V(W$ or $Z$ ) and exploiting the leptonic decays (to $\ell v_{\ell}, \ell \ell$ or $v_{\ell} v_{\ell}$, with $\ell=e$ or $\mu$ ) of the $V$. A lot of efforts have been made in improving the resolution of the $b \bar{b}$ invariant mass $\left(m_{b b}\right)$. Fig. 1 shows the invariant mass of the two $b$-jets from the simulation of the process $p p \rightarrow Z H+X, Z H \rightarrow \ell \ell b b$. The result obtained using the standard jet-

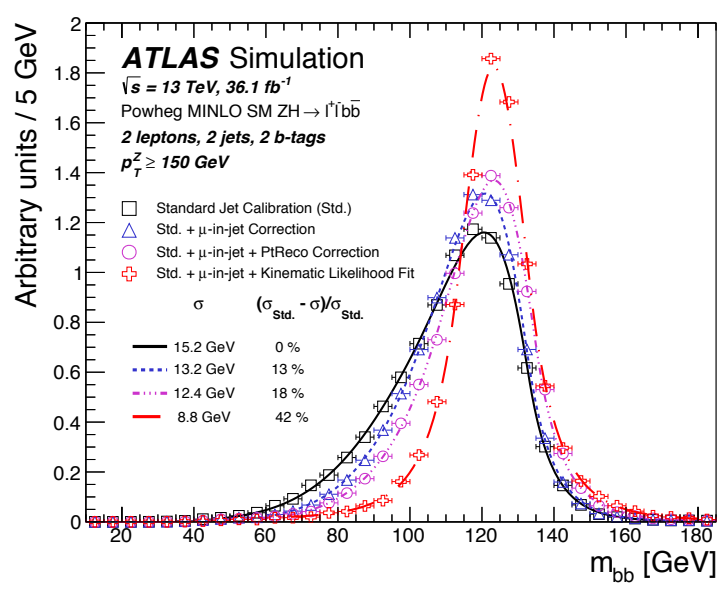

Figure 1. Comparison of the $m_{b b}$ distributions as additional corrections are applied to the jet energy scale, shown for simulated events of the process $p p \rightarrow Z H+X Z H \rightarrow \ell \ell b b$ [2].

calibration can be substantially improved including further corrections to account for $\mu$ within $b$-jets and exploiting a kinematic likelihood fit for the decay channel $\mathrm{ZH} \rightarrow \ell \ell b b$ where the final state can be fully reconstructed and the transverse momentum $\left(p_{T}\right)$ of the $b$-jet pair is constrained by the $p_{T}$ of the lepton pair. In order to extract the signal from the large background, a multivariate analysis approach and in particular a boosted decision tree (BDT) was employed. Another important peculiarity of this process is the possibility of a direct cross-check using the analogous process with a $Z \rightarrow b b$ replacing the $H$ in the final state. Applying the analysis scheme to this process ( $p p \rightarrow V Z+X, V \rightarrow \ell v, \ell \ell, v v$, $Z \rightarrow b b$ ) a signal significance of $5.8 \sigma$ (expected $5.3 \sigma$ ) and a signal strength compatible with SM expectations within $1 \sigma$ are obtained, providing an important validation to the BDT analysis. Fig. 2 shows the BDT output (left plot) and the signal strengths (right plot) for the signal process. The results are compatible with SM expectation within $1 \sigma$ and the signal significance, including Run 1 data, is $3.6 \sigma$ (expected $4.0 \sigma$ ). The result represents the first evidence of hadronic decays of the Higgs boson.

\subsection{Higgs decay to $\gamma \gamma$ and four leptons}

Beside the new results, which improve our knowledge of the Higgs boson properties, important progress has been also made in the di-photon and four-leptons decay channels [3]. Fig. 3 presents a compilation of cross-section measurements for different $\sqrt{s}$ (left plot) and the result of a study aiming to estimate the contribution of the different production channels (right plot). All the results are compatible with the SM expectations within the present uncertainties of the measurements. 

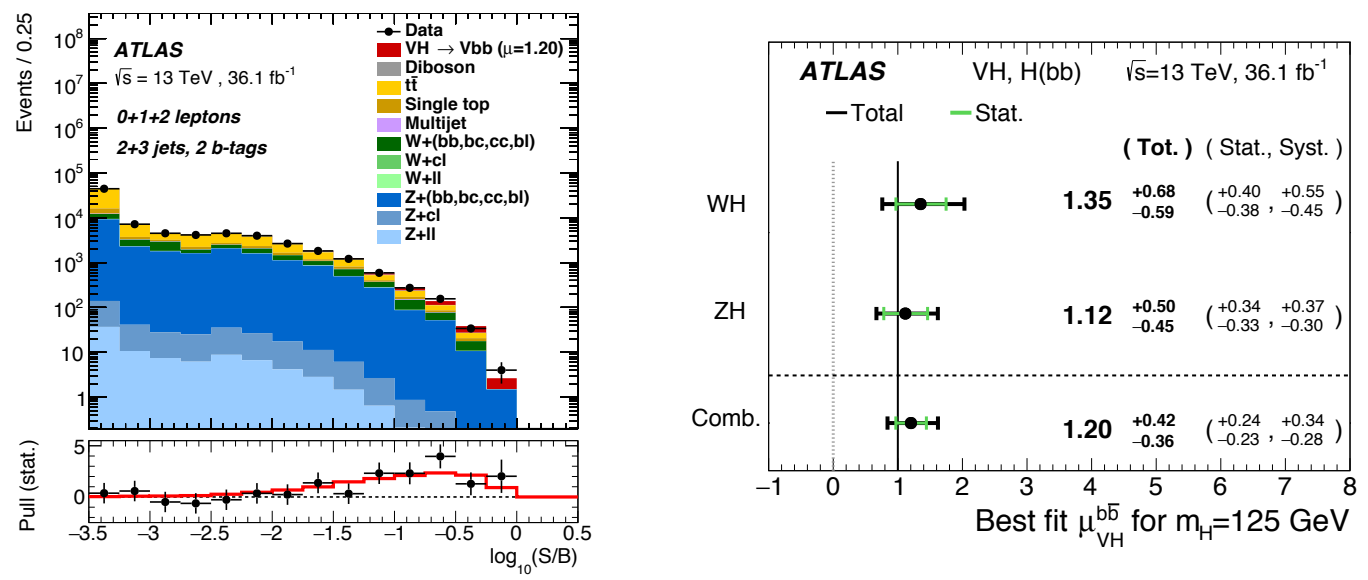

Figure 2. BDT output (left) and signal strengths (right) for the search of the Higgs decay to $b$-quark pairs in associated production with a $W$ or $Z$ boson [2].
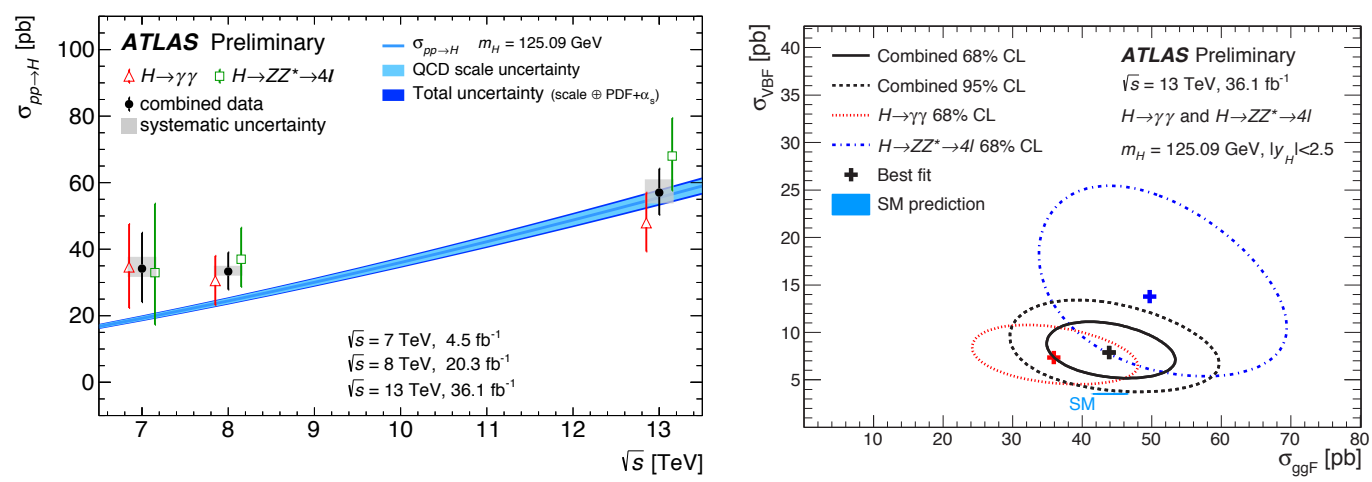

Figure 3. Compilation of Higgs production cross-section measurements for different $\sqrt{s}$ (right) and contribution of the different production processes (left) using $\gamma \gamma$, four-leptons and combined Higgs decay channels [3].

\section{Other Standard Model results}

An impressive number of production channels and final states have been investigated since the beginning of the LHC operations. No significant deviations from SM expectations has been observed up to now. However many channels, such as $s$-channel single-top production, associated production of top pairs and a vector boson, multiple vector bosons production, dibosons production in association with jets, still suffer from large statistical uncertainties and their measurements are expected to be substantially improved in the next couple of years, with the increasing integrated luminosity.

\subsection{Measurement of the $\mathrm{W}$ mass}

The $W$ mass is a fundamental parameter of the SM. Together with the top mass it is tightly related to the Higgs mass and a precise measurement of this parameter allows for crucial consistency tests of the 
electroweak (EW) theory. The measurement is challenging mainly due to the modelling uncertainties of the production process, which affect the $W p_{T}$ modelling and has a direct impact on the mass measurement in the leptonic decay. The ATLAS measurement has been performed in the leptonic decay channel $(W \rightarrow \ell v)$ and restricted to a well understood data-set collected at $\sqrt{s}=7 \mathrm{TeV}$ and corresponding to an integrated luminosity of $4.6 \mathrm{fb}^{-1}$ [4]. One of the key ingredients of the analysis has been the $W$ recoil calibration, performed using $Z \rightarrow \ell \ell$ data and transferring the calibration from the $Z$ to the $W$ production process. The results are summarized in Fig. 4 . The plot on the left shows the comparison between the ATLAS measurement and the LEP and Tevatron ones. The ATLAS estimation of the $W$ mass, $m_{W}=(80.370 \pm 0.019) \mathrm{GeV}$, has a total uncertainty similar to the CDF one, which is the best single experiment result to date. The right plot presents a test of the EW predictions comparing the allowed regions in the $m_{W}$ vs $m_{\text {top }}$ plane for the ATLAS measurements and for an EW fit without $m_{W}$ and $m_{\text {top }}$ as inputs. Fair agreement, within the present uncertainties, is observed between data and SM predictions; further progress will require a substantial improvement in the modelling of the $W$ production.
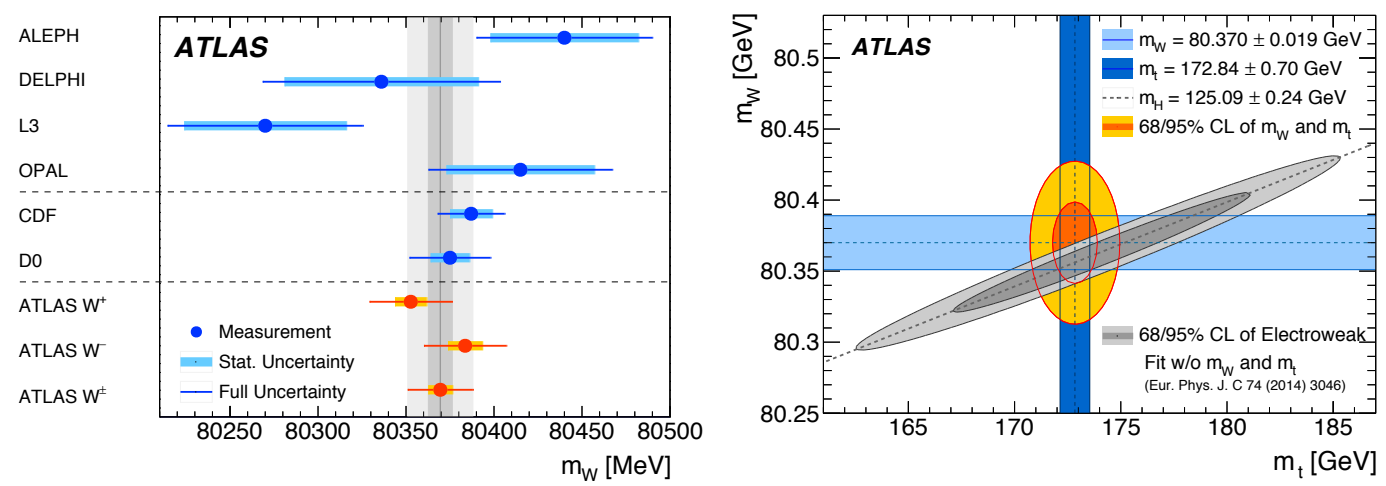

Figure 4. Comparison of the ATLAS measurement with the LEP and Tevatron experiments for the $W$ mass measurement (left). Consistency test for the EW theory in the $m_{W}-m_{\text {top }}$ (right) [4].

\subsection{Top physics}

Top-quarks can be produced at the LHC in pairs or singly via strong or EW interactions, respectively. Fig. 5 shows a compilation of cross-section measurements in different final states and at different $\sqrt{s}$ for top-pair (left plot) and single-top (right plot) production. For the top-pair the overall precision of the measurement is $\sim 4 \%$, at the same level of the theoretical predictions computed at NNLO+NNLL. For the single-top the uncertainty for the dominant $t$-channel production is $\sim 7 \%$. A good agreement with SM expectations is observed in all cases.

The large production cross section of top-quark pairs $(t \bar{t})$ via strong interactions, allows for precise test of QCD predictions through the analysis of differential distributions. A lot of studies have been performed comparing the measured distributions of relevant kinematic variables with the QCD predictions, both at the particle-level (measurements restricted to a phase-space region close to the detector acceptance) and at the parton-level (measurements estrapolated to the whole phase-space). Fig. 6 presents a couple of examples for the $p_{T}$ of the $t \bar{t}$ system in the lepton+jets channel at $\sqrt{s}=13$ $\mathrm{TeV}$ [6] (left plot) and the $p_{T}$ of the lepton in the di-lepton (opposite sign $e \mu$ pairs) channel at $\sqrt{s}=8$ 

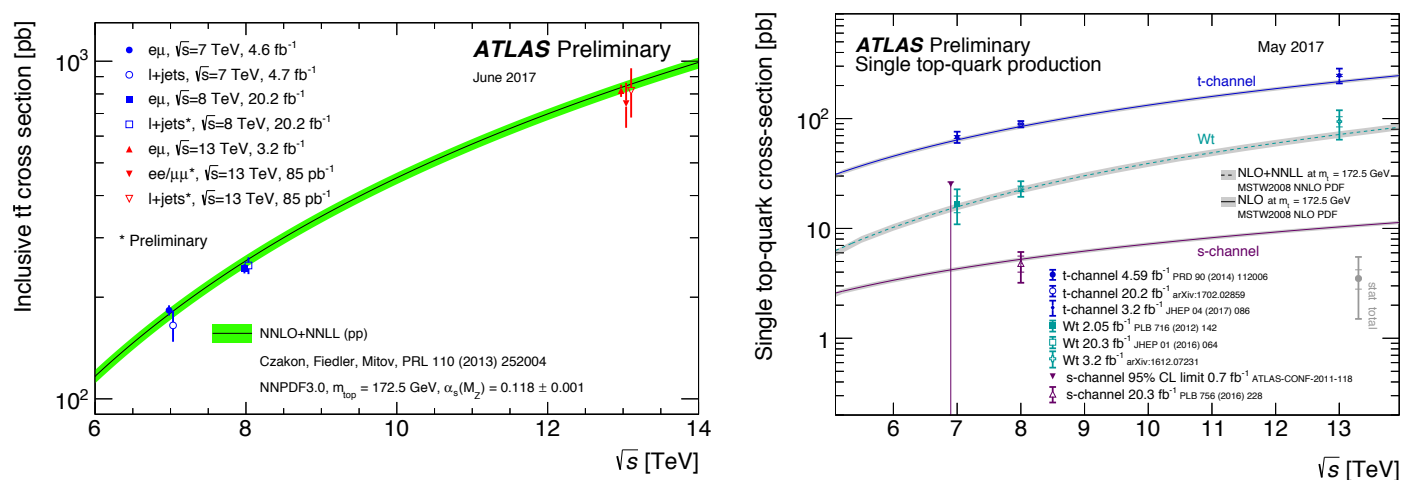

Figure 5. Compilation of top-quark production cross section at different $\sqrt{s}$ compared with SM predictions for top-pair (left) and single-top (right) production [5].

TeV [7] (right plot). The plots show normalized differential cross sections at particle-level compared with several Monte Carlo predictions. These measurements have typical precisions in the range $1-10 \%$ and, beside providing precision test of the QCD predictions, can be used in the tuning of the MC parameters and to extract insights on the gluon parton distribution function at $x \sim 10^{-2}$. Top-pair
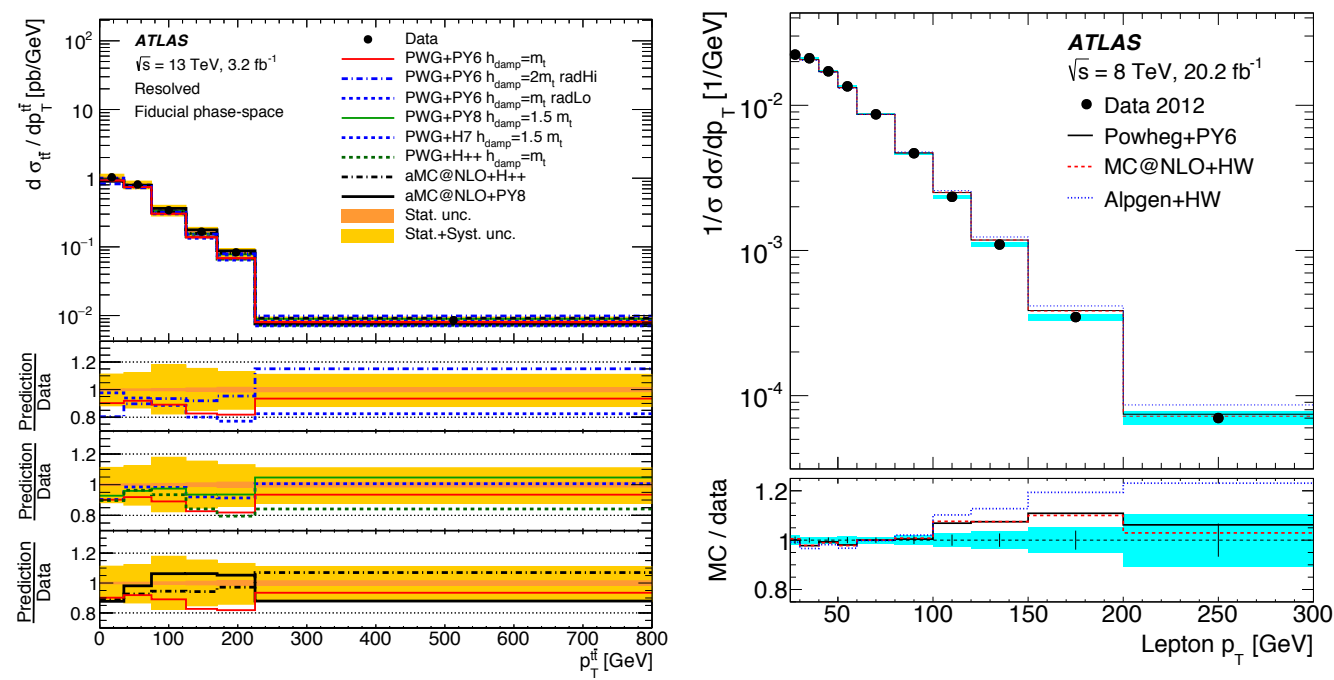

Figure 6. Measurement of the normalized differential cross section for the $p_{T}$ of the $t \bar{t}$ in the lepton+jets channel at $\sqrt{s}=13 \mathrm{TeV}$ (left) [6] and the $p_{T}$ of the lepton in the di-lepton (opposite sign $e \mu$ pair) channel $\sqrt{s}=8 \mathrm{TeV}$ (right) [7]. The measurements have been corrected at the particle-level for detector effects and are compared with several MC predictions.

cross-section measurements can also be used to extract the pole mass of the top quark $\left(m_{t}^{\text {pole }}\right)$ which is expected to differ by $O(1 \mathrm{GeV})$ from the Monte Carlo mass evaluated from the top-quark decay products. In particular a measurement of $m_{t}^{\text {pole }}$ was performed with a simultaneous fit to eight differential 
distributions in the di-lepton channel [7], using a fixed order QCD NLO prediction as implemented in MCFM [8]. Fig. 7 shows a compilation of different measurements of $m_{t}^{\text {pole }}$ at Tevatron and LHC, the ATLAS measurement performed in the di-lepton channel is the most precise to date.

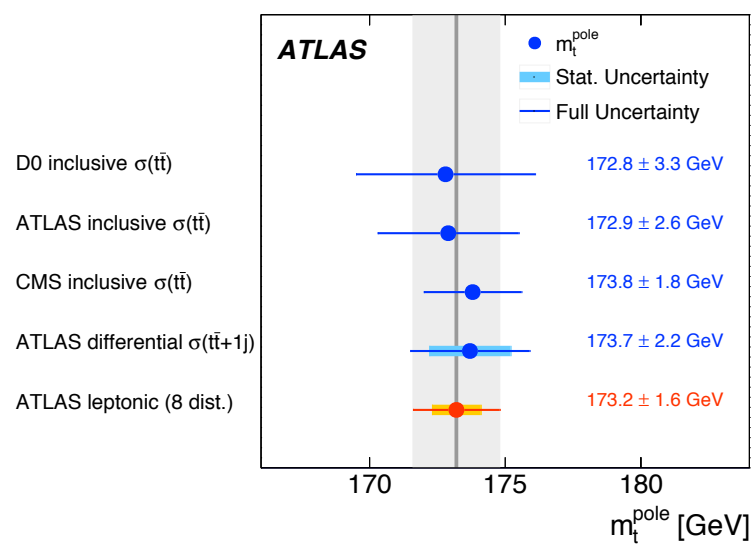

Figure 7. Compilation of $m_{t}^{\text {pole }}$ measurements from different LHC and Tevatron experiments [7].

The excellent performance of the LHC allows the experiments to start a campaign of studies of rare $S M$ processes. The associated production of a top-quark and a $Z$ boson is one interesting example and allows to probe the $W W Z$ and the $t Z$ couplings in a single process, as shown in Fig. 8. The ATLAS
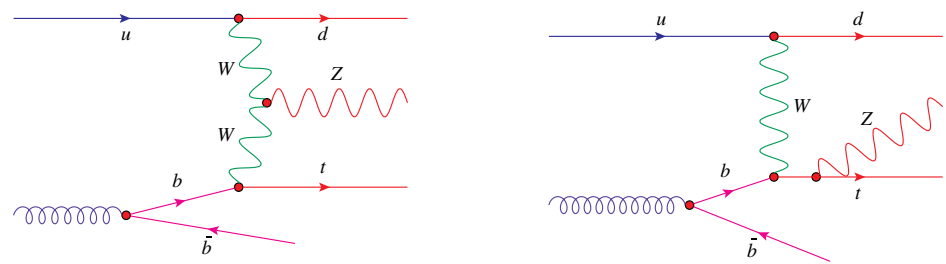

Figure 8. Example of Feynman diagrams of the lowest-order amplitudes for the associated production of a topquark and a $Z$ boson. The largest contributing amplitude to the cross section is shown in the left diagram where the $Z$ boson is coupled to the $W$ boson while the right diagram shows one of the four diagrams with radiation off a fermion [9].

experiment performed the study of this process using the full data-set collected at $\sqrt{s}=13 \mathrm{TeV}$ in 2015 and 2016 and looking for final states with three leptons and two jets, one of which was requested to be tagged as originating from a $b$-quark [9]. The analysis is challenging due to the small cross section of the process and the large background coming from standard processes, such as $t \bar{t}, Z+$ jets, and diboson production. A neural network was used in order to improve the signal to background separation (see Fig. 9). The observed signal significance is $4.2 \sigma$ (expected $5.4 \sigma$ ) and the estimated cross section is $\sigma_{t Z}=620 \pm 170$ (stat.) \pm 140 (syst.) fb, in good agreement with the SM prediction. 


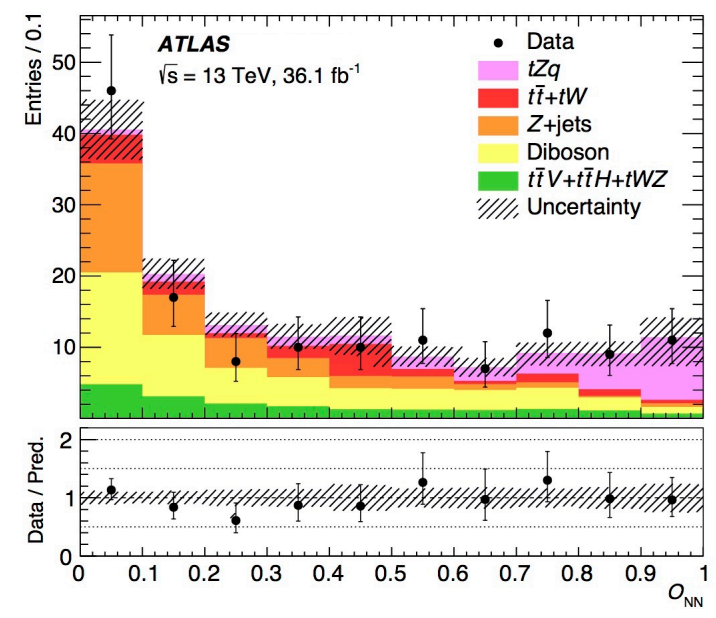

Figure 9. Post-fit neural-network output distribution. Signal and backgrounds are normalised to the expected number of events after the fit. The uncertainty band includes both the statistical and systematic uncertainties as obtained from the fit [9].

\section{Searches for beyond SM physics}

In the search for possible new physics signals a large number of models and final states have been investigated. The sensitivity for beyond SM physics has been largely enhanced by increasing the collision $p p$ energy to $\sqrt{s}=13 \mathrm{TeV}$. At the moment typical mass limits for generic new heavy particles are in the range $2-10 \mathrm{TeV}$, depending on the models and final states considered while the limits for the scale of contact interactions extends up to $40 \mathrm{TeV}$. In the dedicated searches for supersymmetry (SUSY), limits beyond $2 \mathrm{TeV}$ for the gluino mass has been reached for the first time.

\subsection{Searches for resonances decaying to leptons}

A search for new resonances, $Z^{\prime}$ or $W^{\prime}$ like, leading to di-lepton or lepton + missing transverse energy $\left(E_{T}^{\text {miss }}\right)$ final states has been performed using the full $\sqrt{s}=13 \mathrm{TeV}$ data-set collected in 2015-2016 [10, 11]. No significant excesses have been observed with respect to SM predictions, as shown in Fig. 10 for the invariant mass in the di-muon channel (left plot) and the transverse mass in the electron channel (right plot). Limits at 95\% confidence level (CL) for narrow resonances have been extracted for various new physics scenarios (see Fig.11). For the sequential SM (SSM), which assumes heavy partners of the SM weak bosons with the same couplings of the $Z$ and $W$, limits of $4.5 \mathrm{TeV}$ and 5.1 $\mathrm{TeV}$ have been observed for $M_{Z^{\prime}}$ and $M_{W^{\prime}}$, respectively.

\subsection{Searches for dark matter}

Generic searches for dark matter (DM), assumed to behave as weakly interactive massive particles (WIMPS), have been performed looking for topologies with a single high- $p_{T}$ object (jet, photon, $Z$, $H$ ) and $E_{T}^{\text {miss }}$. Indeed, in order for DM to be produced at LHC, the mediator of the production process 

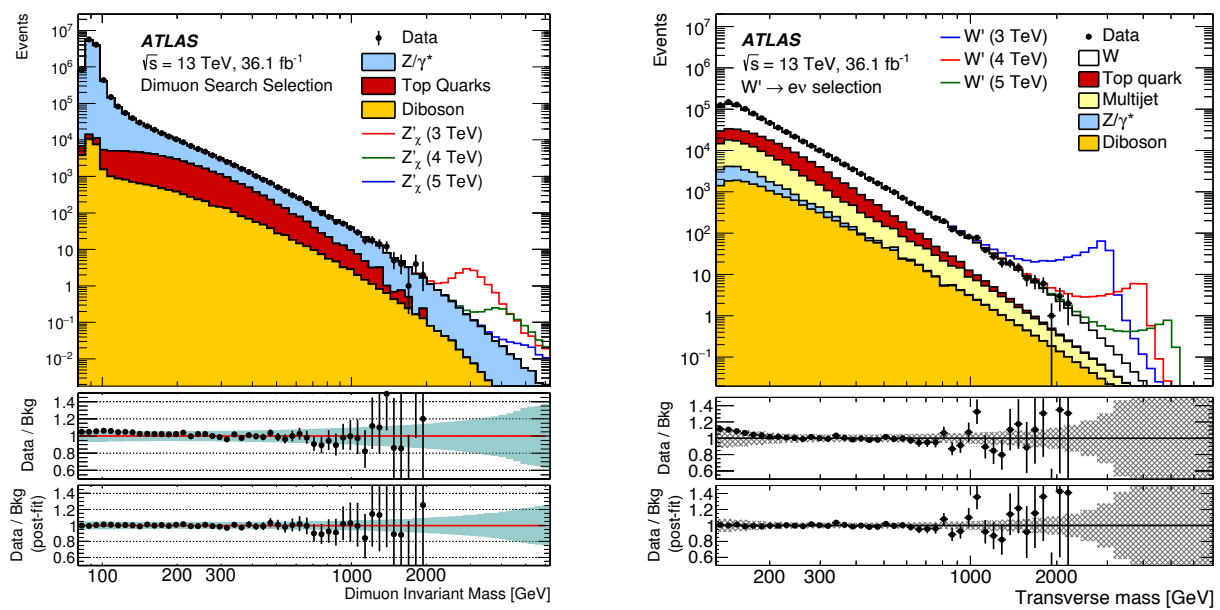

Figure 10. Distribution of dimuon reconstructed invariant mass (left) [10] and transverse mass in the electron channel (right) [11], for data and the SM background estimates as well as their ratio before and after the fit performed to adjust the expected background. Selected signals are also overlaid.
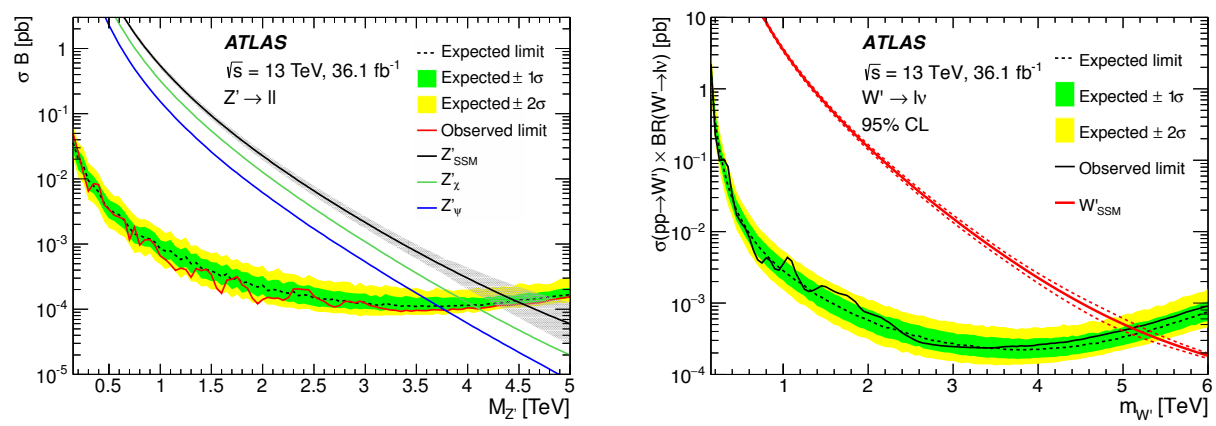

Figure 11. Observed (solid line) and expected (dashed line) upper limits on cross section times branching ratio $(\sigma \times \mathrm{BR})$ as a function of the SSM $Z^{\prime}$ (left) [10] and $W^{\prime}$ (right) [11] boson mass in the combined electron and muon channels. The $1 \sigma$ (green) and $2 \sigma$ (yellow) expected limit bands are also shown. The predicted $\sigma \times$ BR for $\operatorname{SSM} Z^{\prime}$ and $W^{\prime}$ production is also shown.

should couple to quarks or gluons in the initial state ${ }^{1}$ and to the DM and a standard object, which recoils against the invisible WIMP in the final state. The phenomenology is very rich, depending on the mass of the mediator and of the DM and on the value and type of the couplings. An example of model dependent $95 \%$ CL limits in the DM vs mediator mass plane, for a leptophobic axial-vector mediator and for different final states, is shown in the left plot of Fig. 12. The right plot of Fig. 12 presents the same constraints converted in the spin-independent WIMP-nucleon scattering cross section vs DM mass plane, for a direct comparison with experiments performing direct DM searches. The ATLAS

\footnotetext{
${ }^{1}$ This means that also dijet production can be induced by this mediator and the study of these final states is also very effective in probing DM models.
} 
limits have a weak dependence on the DM mass and improve on the direct DM searches for low mass values, up to a few $\mathrm{GeV}$.
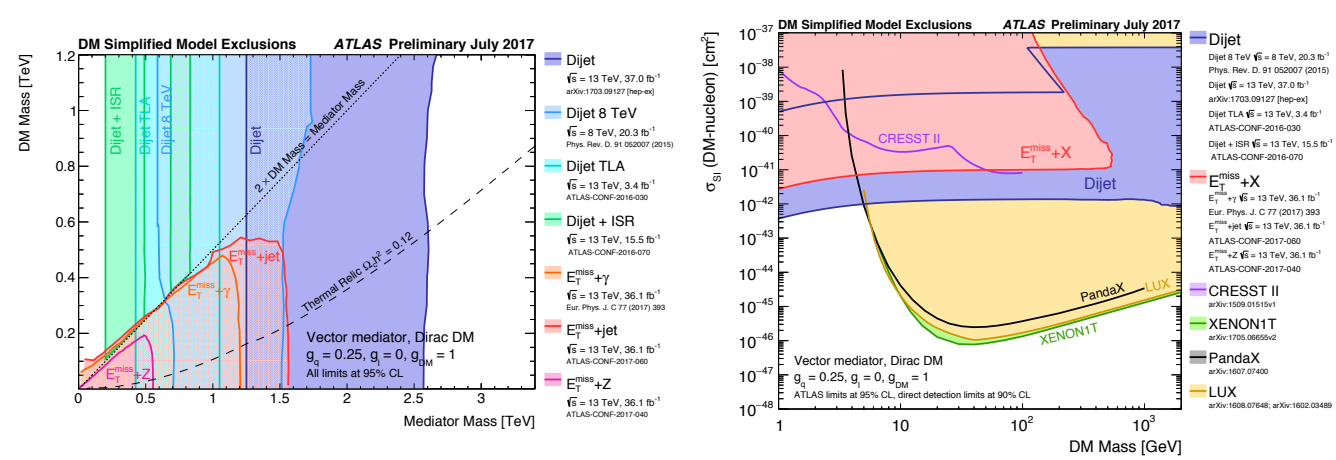

Figure 12. Limits for different ATLAS searches in the mediator vs DM mass plane, for a leptophobic axial-vector mediator and fixed couplings to quarks and DM (left). The same constraints have been converted in limits on spin-independent WIMP-nucleon scattering cross section as a function of DM mass, for a comparison with direct $\mathrm{DM}$ searches (right). The single dijet and $E_{T}^{\mathrm{miss}}+\mathrm{X}$ exclusion region represents the union of exclusions from all analyses of that type [12].

\subsection{SUSY searches}

A search for light squarks and gluinos have been performed exploiting final states with multi-jets and $E_{T}^{\text {miss }}$ and using the full $\sqrt{s}=13 \mathrm{TeV}$ data-set collected in 2015 and 2016. The study looks for pair production of gluinos or squarks decaying to jets and charginos, with the subsequent decay of the charginos to $W$ and neutralinos [13]. The left plot of Fig. 13 shows the inclusive effective mass ${ }^{2}$, $m_{\mathrm{eff}}\left(\right.$ incl.), in the six-jets selection, where $m_{\mathrm{eff}}$ (incl.) is evaluated using all the jets with $p_{T}>50 \mathrm{GeV}$. No significant excess with respect to the SM expectations have been found and limits beyond $2 \mathrm{TeV}$ have been set for the gluino mass, as shown by the right plot of Fig. 13 which presents a compilation of $95 \%$ CL limits in the neutralino vs gluino mass plane, also including the results of other analysis exploiting different final states.

The third generation squarks, sbottom $(\tilde{b})$ and stop $(\tilde{t})$, in natural SUSY models are expected to be substantially lighter than the squark of the first and second generations. Consequently, the searches for direct pair production of $(\tilde{b})$ and stop $(\tilde{t})$ are of primary interest and have been updated exploiting the full available data-set. Fig. 14 presents the constraints for $\tilde{b}$ (left plot) and $\tilde{t}$ (right plot) in the neutralino vs squark mass plane. The $\tilde{b}$ analysis exploits the decay $\tilde{b} \rightarrow b \chi^{0}$ looking for final state with $b$-jets and $E_{T}^{\text {miss }}$ [15], while the $\tilde{t}$ results include studies of different final states with 0,1 or 2 leptons. In both cases the limits extend up to masses close to $1 \mathrm{TeV}$ with a significant improvement with respect to the Run 1 limits at $\sqrt{s}=8 \mathrm{TeV}$.

\footnotetext{
${ }^{2}$ The effective mass $m_{\mathrm{eff}}\left(N_{j}\right)$ is defined, for events selected requiring at least $N_{j}$ jets, as the scalar sum of the $p_{T}$ of the $N_{j}$ jets and the $E_{T}^{\text {miss }}$. Due to the high mass scale expected for the SUSY models studied in this search it is a powerful discriminant between signal and SM background.
} 

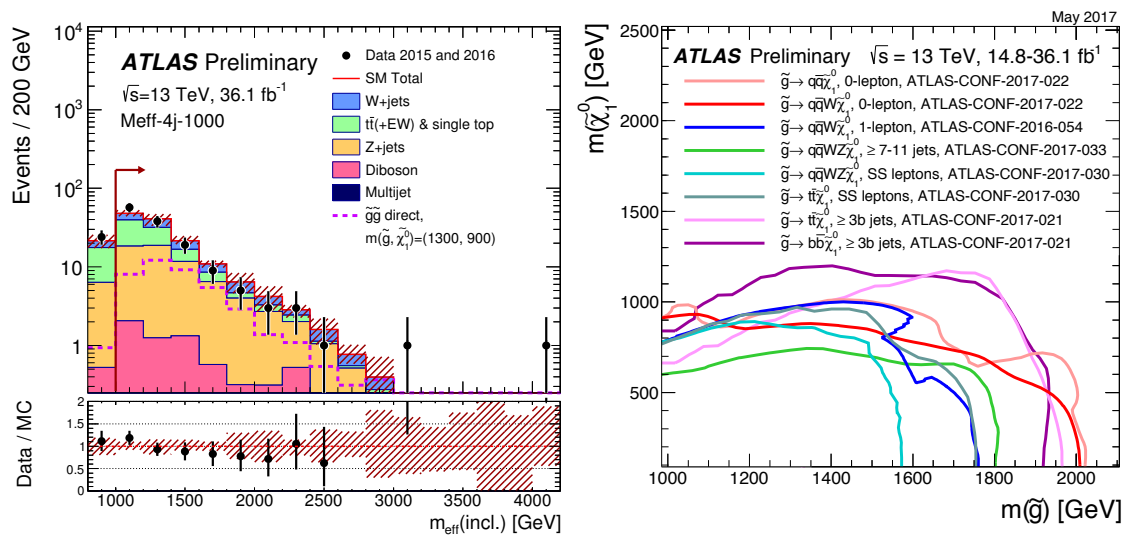

Figure 13. Observed $m_{\text {eff }}$ (incl.) distribution for the six-jets selection (left) [13]. The hatched error bands denote the combined experimental and MC statistical uncertainties. The arrows indicate the cut values applied in the analysis. Expected distributions for benchmark signal model points, normalized to NLO+NLL cross section times integrated luminosity, are also shown for comparison. The right plot shows a compilation of ATLAS limits in the neutralino vs gluino mass plane, which includes results from several analysis exploiting different final states [14].
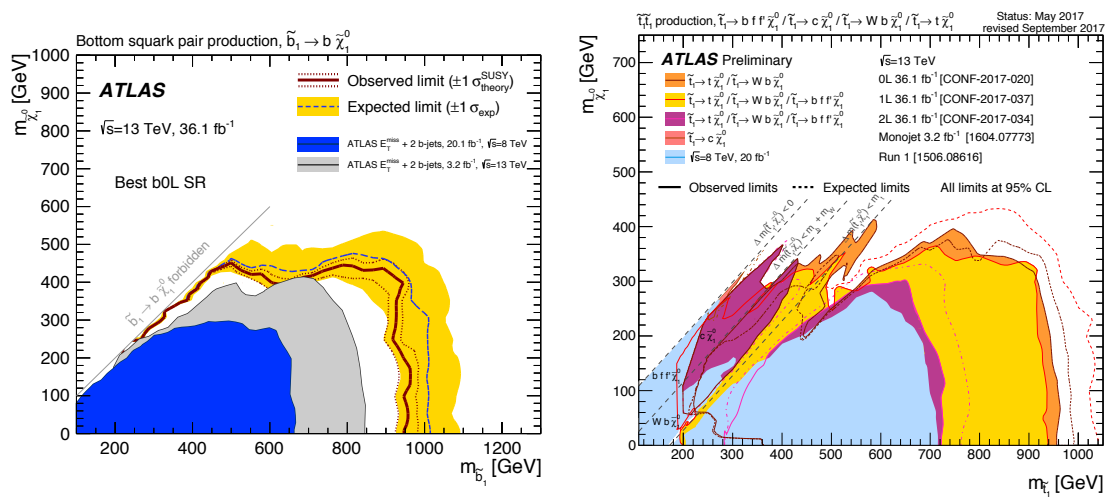

Figure 14. Constraints for $\tilde{b}$ (left) [15] and $\tilde{t}$ (right) [14] in the neutralino vs squark mass plane. The results of the Run 1 studies at $\sqrt{s}=8 \mathrm{TeV}$ are also shown.

\section{Heavy lon physics}

The ATLAS experiment was designed as a general purpose detector for the study of $p p$ collisions. Nevertheless a well defined program of measurements exploiting heavy ion collisions has been developed and succesfully pursued. A recent interesting result is the study of light-by-light scattering (LbyL, $\gamma \gamma \rightarrow \gamma \gamma$ ), in $5 \mathrm{TeV}$ ulta-peripheral (UP) $\mathrm{Pb}-\mathrm{Pb}$ collisions. LbyL scattering was observed in photon scattering with Coulomb field of nuclei or in the photon splitting due to the interaction with an external field. Nevertheless elastic LbyL, also related to vacuum polarization, has never been observed up to now. In UP heavy ion collisions the strong interaction does not play a role and the strong electromagnetic field produced by the scattering nuclei can be treated as a beam of quasi-real photons. 
The cross section for the reaction $\mathrm{Pb}+\mathrm{Pb}(\gamma \gamma) \rightarrow \mathrm{Pb}^{*}+\mathrm{Pb}^{*} \gamma \gamma$ can be calculated by convolving the respective photon flux with the elementary cross section for the process $\gamma \gamma \rightarrow \gamma \gamma$. Since the photon flux associated with each nucleus scales as $Z^{2}$, the cross section is strongly enhanced with respect to $p p$ collisions. The analysis has been performed using $\mathrm{Pb}-\mathrm{Pb}$ collisions at $\sqrt{s}=5 \mathrm{TeV}$ corresponding to an integrated luminosity of $480 \mu \mathrm{b}^{-1}$ and looking for di-photon final states in UP collisions [16]. Fig. 15 shows the acoplanarity of the two photons (left plot) and the invariant mass of the photon pairs (right plot) after a cut on the acoplanarity to improve the signal to background ratio. A signal significance of $4.4 \sigma$ (expected $3.8 \sigma$ has been obtained and the measured fiducial cross section $\sigma_{\text {fid }}=70 \pm 24$ (stat.) \pm 17 (syst.) $\mathrm{nb}$ is in good agreement with SM predictions.
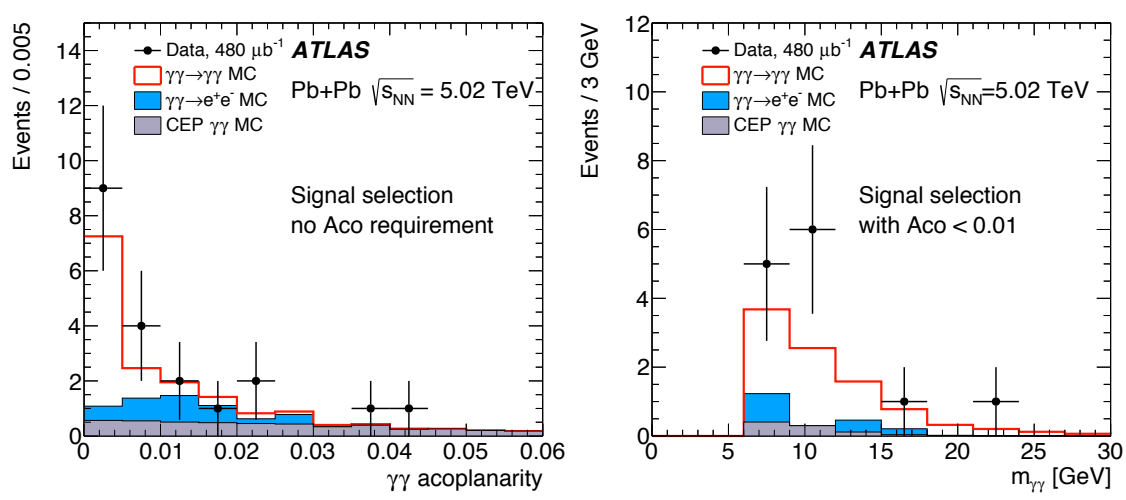

Figure 15. Acoplanarity of the two photons (left) and invariant mass of the photon pairs (right). The data are compared with the background and signal predictions [16].

\section{Conclusions}

An impressive amount of groundbreaking results have been produced by ATLAS using the $p p$ and heavy-ion collision data collected so far, only a subset has been presented in this report. These results contributed to a significant improvement in our understanding of the fundamental laws of Nature at the microscopic level. The SM has been probed through a plethora of measurements with unprecedented precision, significant steps forward have been done towards a better comprehension of the Higgs sector, and searches for new physics, especially exploiting the Run 2 LHC data, have been pushed far beyond the reach of the pre-LHC era. This impressive list of outstanding results couldn't have been obtained without the exceptional performances and reliability of the LHC operations. The 2017 run is shaping up to be another year of record for LHC, possible relevant updates could be available soon.

\section{References}

[1] ATLAS Collab., JINST 3, S08003, (2008).

[2] ATLAS Collab., arXiv:1708.03299[hep-ex], submitted to JHEP

[3] ATLAS Collab., ATLAS-CONF-2017-047, cds.cern.ch/record/2273854

[4] ATLAS Collab., arXiv:1701.07240[hep-ex], submitted to EPJC

[5] atlas.web.cern.ch/Atlas/GROUPS/PHYSICS/CombinedSummaryPlots/TOP/ 
[6] ATLAS Collab., arXiv:1700.00727[hep-ex], submitted to JHEP

[7] ATLAS Collab., arXiv:1709.09407[hep-ex], submitted to EPJC

[8] J. M. Campbell and R. K. Ellis, Nucl. Phys. Proc. Suppl. 205-206, 10, (2010), arXiv:1007.3492[hep-ph]

[9] ATLAS Collab., arXiv:1710.03659[hep-ex], submitted to PLB

[10] ATLAS Collab., arXiv:1707.02424[hep-ex], submitted to JHEP

[11] ATLAS Collab., arXiv:1706.04786[hep-ex], submitted to EPJC

[12] atlas.web.cern.ch/Atlas/GROUPS/PHYSICS/CombinedSummaryPlots/EXOTICS/

[13] ATLAS Collab., ATLAS-CONF-2017-022, cds.cern.ch/record/2258145

[14] atlas.web.cern.ch/Atlas/GROUPS/PHYSICS/CombinedSummaryPlots/SUSY/

[15] ATLAS Collab., arXiv:1708.09266[hep-ex], submitted to JHEP

[16] ATLAS Collab., Nature Physics, 13, 852, (2017), arXiv:1702.01625[hep-ex] 